\title{
Atlante dei siti fortificati della provincia di Viterbo, Italia (X-XV secolo). Fonti e metodi per la ricostruzione della rete insediativa bassomedievale
}

Atlas of fortified settlements in the province of Viterbo, Italy (tenth-fifteenth centuries). Sources and methods for the reconstruction of the late-medieval settlement network

\author{
Maurizio Toscano $^{\text {a }}$, Giuseppe Romagnoli ${ }^{\text {b }}$ \\ a Universidad de Granada, Granada, Spain, maurizio.toscano@gmail.com \\ b Università degli Studi della Tuscia, Viterbo, Italy, romagnoli@unitus.it
}

\begin{abstract}
This study addressed the historical phenomenon known as incastellamento, in the area of the current province of Viterbo, from a quantitative and geographical perspective. The time period considered was the tenth-fifteenth century. The paper describes the documentary sources, historical maps, aerial images, past studies and archaeological sources that are available to researchers, and which have been used, in good measure, to reconstruct the fortified settlement network. Moreover, the paper explains the methodologies used to identify, store and geocode the whole dataset, which so far comes to a total of 191 fortified settlements. In conclusion, we discuss the main characteristics of the online atlas, intended as an open and interoperable platform to consult, query and retrieve information from the dataset of late-medieval fortified settlements.
\end{abstract}

Keywords: Middle Ages, fortified settlements, historical gazetteer, data modelling, Web Information System, Linked Open Data, Digital Humanities, geographic information science.

\section{Introduzione}

Ambito territoriale oggetto del presente studio è lo spazio racchiuso all'interno dei confini della provincia di Viterbo, quali risultano dalla corrente suddivisione amministrativa, l'ambito cronologico quello compreso tra i secoli X e XV d.C. ed il campo d'indagine quello dell'impianto di abitati fortificati, conosciuti generalmente dalle fonti medievali come castra o castella. L'area selezionata coincide in buona parte con i limiti del Patrimonio di San Pietro in Tuscia, nella denominazione e delimitazione territoriale assunta nell'XI secolo e conservata fino al XVIII.

Ciò che ha mosso l'indagine non è stato l'interesse per il singolo castello, o per quanto da esso si potesse estrapolare in termini di storia militare, architettonica o urbanistica della provincia, ma per la rete unitaria degli insediamenti nel suo insieme, ricostruita attraverso una serie di fonti e metodologie che descriveremo nei prossimi capitoli. Ciò che muove, infine, la proposta di pubblicazione dell'atlante web dei siti fortificati della provincia di Viterbo è la volontà di rendere pubblici i dati raccolti, espliciti gli strumenti e i metodi utilizzati e riproducibili i risultati delle analisi condotte (Toscano, 2008), secondo i principi della Scienza aperta con Dati Aperti (Machado, 2015) e attraverso la pratica dell'informatica umanistica. 


\section{Fonti per la ricostruzione della rete}

$\mathrm{Si}$ passano qui di seguito in rapida rassegna le principali fonti archeologiche, storico-documentarie e cartografiche utili per l'ubicazione dei siti incastellati e, più in generale, per la ricostruzione della rete dei castelli nell'ambito territoriale esaminato.

\subsection{Le fonti documentarie}

Tra le fonti di matrice ecclesiastica, una posizione preminente spetta alla documentazione dei grandi enti monastici che, a partire dall'altomedioevo, costituirono i principali proprietari fondiari del territorio a Nord di Roma. Se il ruolo di S. Maria di Farfa e di S. Salvatore al Monte Amiata nel territorio altolaziale si riduce notevolmente nell'età dei castelli fino a divenire insignificante, a partire dal X secolo si accresce il ruolo dei grandi monasteri romani. Tra i cartulari editi di chiese e monasteri romani, si possono menzionare quelli dell'Abbazia di San Paolo fuori le Mura (Trifone, 1908-1909), della Basilica Capitolare di S. Pietro in Vaticano (Schiaparelli, 1901-1902), del Monastero di Sant'Anastasio ad Aquas Salvias (Giorgi, 1878), del Monastero di San Silvestro in Capite (Federici, 1899-1900), dell'Abbazia di Sant'Alessio all'Aventino (Monaci, 1904-1905), dei Monasteri dei SS. Cosma e Damiano in Mica Aurea (Fedele, 1898), di S. Maria in Campo Marzio (Carusi, 1948), di Santa Maria in Via Lata (Hartmann, Merones, 1895-1913) e di Santa Maria Nova (Fedele, 1900-1903). A questi importanti nuclei documentari si possono aggiungere le carte di alcuni cenobi umbri, come l'Abbazia di Sassovivo (Cencetti, et al., 1973-1975), e altolaziali.

Rivestono una particolare rilevanza anche i fondi degli enti ecclesiastici viterbesi: le carte del Capitolo della Cattedrale di Viterbo (pubblicate integralmente fino al 1299: Egidi, 1906-1907), delle collegiate di SS. Stefano e Bonifacio di Viterbo (Buzzi, 1988), di S. Angelo in Spatha, S. Sisto e dei Conventi di S. Maria della Verità e della Trinità (Savignoni, 1895).

Per quanto concerne le fonti fiscali e di governo, sono fondamentali per la storia del popolamento il Liber Censuum della Chiesa Romana (Fabre, Duchesne, 1905-1952), i censi e le collettorie della Santa Sede (Theiner, 1861-1862), i registri delle decime, che coprono, con varie soluzioni di continuità dovute al carattere episodico di questo tipo di riscossione, il periodo che va dal 1274 alla prima metà del XIV secolo (Battelli, 1946), ed i registri delle tassazioni del sale, eseguite semestralmente dai gabellieri dello Stato della Chiesa (Tomassetti, 1897). Ritrovata dall'archivista De Rossi a Siena, e per questo conosciuta col nome di "Romano-Senese", ma relativa al territorio a nord di Roma, è invece una tassazione del sale e del focatico pubblicata dal Tomassetti alla fine dell'Ottocento e datata da questo studioso alla metà del XIV secolo. Sempre per il Trecento, sono di notevole interesse anche le relazioni inviate dal Rettore del Patrimonio al Pontefice residente in Avignone (Antonelli, 1895) e i registri del Cardinal Albornoz del 1364 (Fabre, 1887).

Passando alle fonti di matrice comunale, il fondo diplomatico del Comune di Viterbo (comprendente la Margarita Viterbese) costituisce naturalmente una fonte di primario interesse per la ricostruzione dei rapporti delle istituzioni comunali con le comunità soggette e con le signorie di castello del territorio (Carbonetti Vendittelli, 1990), così come l'analoga documentazione prodotta dalla città di Orvieto (Fumi 1884) e da Corneto (Supino 1969). Anche per altri centri del Patrimonio di S. Pietro è stato edito un buon numero di fonti civiche, disponibili in varie raccolte. Tra le fonti normative, spiccano gli statuti comunali medievali di Viterbo degli anni 1237-38, 1251-52 e 1469 (Egidi 1930; Buzzi, 2004) e quelli del castello di Fiorentino, una delle comunità soggette tra XIII e XIV secolo al comune viterbese (Egidi, 1910).

Per la ricostruzione della geografia del potere delle grandi famiglie patrizie, i patrimoni meglio documentati sono quelli dei Colonna, degli Orsini e degli Anguillara (Sora, 1906), restando invece quasi priva di documentazione la dominazione feudale della famiglia Normanni-Alberteschi sulla fascia costiera della Tuscia Romana. Sempre riguardo i grandi proprietari terrieri, risultano di grande interesse gli atti pervenutici relativi al passaggio dei beni territoriali all'Ordine di San Giovanni in Gerusalemme dopo la soppressione dei Templari, conservati anch'essi presso l'Archivio Vaticano (Silvestrelli, 1917). 
Si segnalano, infine, le cronache quattrocentesche di Viterbo, compilate, per la parte anteriore al XV secolo, in parte sulla base di fonti più antiche (Egidi, 1901).

\subsection{Le fonti cartografiche e aerofotografiche}

Lo spoglio delle mappe storiche del Lazio (Almagià, 1960; Frutaz, 1972) non è infruttuoso, anche se la cartografia riguardante il territorio regionale inizia a diventare significativa solo a partire dal XVI secolo. La prima carta che riproduce in maniera molto precisa ed estesa buona parte del territorio laziale del Patrimonio di San Pietro in Tuscia è quella redatta dal Bellarmati nel 1536, illustrativa però dei soli centri ancora esistenti all'epoca. Dovendo escludere la splendida carta di Eufrosino della Volpaia del 1547, riferita alla sola Campagna Romana, per ottenere una esauriente descrizione della nostra area dobbiamo attendere gli inizi del XVII secolo, con la redazione dell'atlante del Magini, tra l'altro carente in riferimento alla quantità di insediamenti inseriti. Di maggiore interesse ai nostri fini risultano le carte dell'Oddi, del Mattei e dell' Ameti, con numerosi riferimenti agli insediamenti di età classica e medievale, anche distrutti, ed alla rete viaria dell'epoca di redazione. Sempre riferita al territorio del Patrimonio è la carta redatta nel XVIII secolo dai padri Maire e Boscovich, attualmente conservata presso la Calcografia Nazionale, mentre a piccole porzioni di territorio, ma con una ricca dotazione toponomastica, fanno riferimento quella del Ligustri del 1609, relativa ad una porzione del territorio costiero della Tuscia, e quella dipinta da Pietro da Cortona nella Villa Chigi a Castelfusano.

Il catasto geometrico-particellare dello Stato della Chiesa, prodotto in due serie nei periodi 1815 1835 e 1855-1875, conservate presso gli archivi di Stato di Roma e di Viterbo e note rispettivamente con i nomi di Catasto Pontificio e Catasto Gregoriano, rappresenta la prima rappresentazione completa del territorio metricamente attendibile e conserva il patrimonio toponomastico più dettagliato riferibile alle campagne laziali, oltre ad una preziosa immagine latente dell'uso del suolo, precedente agli sconvolgimenti subiti dal paesaggio a partire dalla prima metà del Novecento.

La cartografia in scala 1:25000, prodotta dall' Istituto Geografico Militare Italiano a partire dal 1875 circa, riveste un importantissimo ruolo come fonte d'indicazioni toponomastiche ormai scomparse o comunque non riportate nella più recente cartografia regionale in scala 1:10000, tanto da poter essere considerata ormai alla stregua di una "cartografia storica".

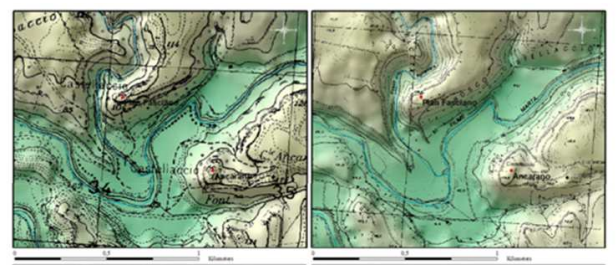

Fig. 1. Ricchezza toponimica I.G.M. a confronto con le carte regionali: esempi di Ancarano e Pian Fasciano.

Un ultimo accenno meritano le coperture aerofotografiche del territorio, basilari per la localizzazione di molti degli insediamenti scomparsi del territorio altolaziale. Quella realizzata sull'intera provincia di Viterbo tra il 1938 e il 1940 ai fini della realizzazione del Catasto Italiano, nota come "Ala Littoria" e attualmente conservata presso l'Archivio di Stato di Viterbo, rappresenta da questo punto di vista una fonte di eccezionale interesse, sia per la scala delle riprese, sia per la sua cronologia, riproducendo un'immagine del paesaggio altolaziale antecedente al boom edilizio ed alle grandi trasformazioni agrarie del secondo dopoguerra.

\subsection{I principali studi storici}

Pur arrivando solo a lambire il territorio altolaziale, il lavoro storico-topografico di Giuseppe Tomassetti sulla Campagna Romana medievale e moderna rappresenta tuttora una importante base documentaria per la ricostruzione degli assetti del territorio a Nord di Roma (Tomassetti, 19101956; Carocci, Vendittelli, 2004).

La pubblicazione di P. Toubert sul Lazio medievale (Toubert, 1973), sebbene incentrata sulla Sabina, ha impresso una vera e propria svolta alla ricerca storica sul fenomeno dell'incastellamento 
nel Lazio, fornendo l'impulso per nuove ricerche di carattere sia storico che archeologico. Nel quarantennio successivo sono mancate sintesi sul territorio altolaziale, sebbene siano apparsi numerosi studi di carattere topografico o storico-documentario, in particolare sull'area circostante il centro urbano di Viterbo tra VIII e XI secolo (Lanconelli, 1992), sul territorio di Sutri (Vendittelli, 2008) e sull' area dei Monti della Tolfa (Vallelonga, 2012).

I repertori dei siti medievali del Lazio curati da G. Silvestrelli (Silvestrelli, 1914), da E. Martinori (Martinori, 1932-1934) e quelli, più recenti, delle sedi umane abbandonate del Patrimonio di San Pietro tra X e XVI secolo (Conti, 1980) e dei siti fortificati del Lazio (Istituto Italiano dei Castelli, 1985), conservano tutt'oggi una certa utilità ai fini di una prima valutazione quantitativa del fenomeno dell'incastellamento, sebbene siano esclusivamente basati sullo spoglio delle fonti edite. Informazioni utili all'ubicazione dei centri incastellati altolaziali solo reperibili anche in due repertori toponomastici sulla diocesi di Orte e sulla provincia di Viterbo (Lungo, 1998, 1999).

Vanno infine ricordate alcune opere di storiografia municipale, anche datate, quali quelle che hanno avuto ad oggetto le vicende medievali di Viterbo (Pinzi, 1887-1913; Signorelli, 19071969) e di Tuscania (Campanari, 1856).

\subsection{Le ricerche archeologiche}

Evitando di passare in rassegna in modo sistematico le indagini archeologiche (per le quali si rimanda alle sintesi in Molinari, 2010; Lanconelli, Romagnoli, 2018), si ricorda che le ricognizioni territoriali nell'Etruria Meridionale vantano una lunga tradizione, che risale indietro fino al grande progetto post-unitario della Carta Archeologia d'Italia (Gamurrini, et al., 1972), ed hanno contribuito in modo decisivo a scrivere la storia dei paesaggi altolaziali per l'età post-classica. Il progetto del South Etruria Survey, condotto dalla British School at Rome tra gli anni Cinquanta e Settanta del secolo scorso, ha comportato prospezioni di carattere sistematico in un'ampia regione a Nord di Roma, corrispondente ai territori storici di Veio, Falerii, Sutri e Capena (Duncan, 1958; Jones, 1962-1963; Wickham, 1978-1979; Potter,
1985), ed è stato integrato da sondaggi di scavo in alcuni siti campione (Mallet, Whitehouse, 1967; Cameron et al., 1984; Christie, 1991; Potter, King, 1997). I dati relativi al periodo compreso tra la tarda antichità e i secoli centrali del medioevo sono stati sistematicamente riesaminati nell' ambito del Tiber Valley Project, coordinato dalla British School at Rome a partire dal 1997 (Patterson, 2004; Coarelli, Patterson, 2004). Nel quadro delle ricognizioni territoriali si menzionano anche le recenti indagini condotte nell'ambito del progetto Carta Archeologica d'Italia, che mostrano un'apertura -se non uniforme almeno costante- alle emergenze archeologiche di età post-antica (Milioni, 2002; Scardozzi, 2004).

Per il suo carattere di completezza, il repertorio degli scavi e delle scoperte archeologiche -prevalentemente di età etrusca e romana- effettuate in Etruria meridionale tra il 1939 e il 1975 nel territorio della Soprintendenza Archeologica dell'Etruria Meridionale può rivelare notizie di interventi su singoli centri, altrimenti difficilmente reperibili (Sommella Mura, 1969; Brunetti Nardi, 1972-1981).

Gli insediamenti rurali medievali dell'entroterra di Civitavecchia e della Teverina viterbese sono stati oggetti di specifiche indagini tematiche condotte nell' ambito dei progetti di scavo delle città medievali di Leopoli-Cencelle presso Civitavecchia (Nardi Combescure, 2002) e di Ferento presso Viterbo (Romagnoli, 2006). Tra gli scavi archeologici in centri incastellati, si possono menzionare almeno quelli condotti a Castel d'Asso presso Viterbo (Güll, et al., 2001) e a Pian Fasciano presso Viterbo (Fronti, Romagnoli, 2007).

Anche se non afferente ad un progetto formalizzato, ma comunque raggruppabile in un insieme coerente, un'ampia e interessante casistica, in particolar modo frutto di tesi di laurea e di specializzazione, è offerta dagli studi promossi negli ultimi anni nell'ambito delle cattedre di Archeologia Medievale delle Università di Roma la Sapienza e della Tuscia, che hanno sviluppato filoni di studi sulla facies rupestre dei siti incastellati (Minicis, 2003-2011) e sull'analisi delle architetture e sulle tecniche costruttive delle strutture fortificate (Andrews, 1978; Chiovelli, 2007). 


\section{Metodologie di gestione, localizzazione e pubblicazione web degli insediamenti}

\subsection{L'architettura dei dati}

Per la raccolta strutturata delle informazioni si è creata, in ambiente GIS, una banca dati relazionale, a partire da un modello dati specifico realizzato per registrare il fenomeno dell'incastellamento, che comprende anche i vincoli d'integrità e i vocabolari necessari alla normalizzazione del linguaggio. In particolare, presentano thesauri i seguenti attributi: comune, tipologia sito, affidabilità (cronologia e georeferenziazione), condizioni attuali e detentore. Completano la scheda sito i campi: toponimi, prima ed ultima menzione, attestazioni pre-castrensi, notizie documentarie e osservazioni. Si sono omessi, in fase di elaborazione del modello dati, gli attributi ottenibili tramite topologia spaziale: coordinate, idrografia, geologia e indice topografico (TPI). Le due tabelle principali sono dedicate l'una ai siti fortificati e l'altra alla relativa bibliografia. Per permettere di gestire l'evoluzione nel tempo della tipologia insediativa e della proprietà, alla tabella sito è stata collegata, attraverso una chiave esterna, la tabella detentori.

\subsection{La mappatura}

Il criterio che si è scelto di seguire nell'individuazione degli abitati da schedare è stato quello della presenza in essi, almeno in una determinabile fascia temporale, di strutture fortificate. Si sono esclusi i castelli di fondazione tardoantica che non avessero testimonianze storiche successive al 900 d.C., inserendo invece quelli che restano in vita nel periodo successivo.

Per la geolocalizzazione dei toponimi, la fonte primaria è stata la cartografia IGM, alla quale si è affiancata, per approfondire il dettaglio nel posizionamento dei siti, il modello digitale del terreno e la copertura ortofotografica in bianco e nero e a colori dell'intero territorio provinciale. Per la loro natura di siti fortificati, questi insediamenti erano delimitati da una o più cinte murarie $e$, in linea di principio, si sarebbero potuti rappresentare mediante l'uso di poligoni. Ciò avrebbe però escluso dalla rappresentazione tutti quei siti dove l'evidenza materiale della fortificazione manca o è frammentaria, e si è quindi prediletto l'uso della geometria puntuale, applicabile a tutti gli insediamenti. Determinata la geometria da utilizzare, è stato necessario definire un metodo per determinare la localizzazione del punto ${ }^{1}$ : sul cassero/torre, ove visibile nel caso di insediamenti in vita; sul simbolo $::$ che nella cartografia IGM indica la presenza di rovine, nei casi di insediamenti abbandonati; sulla quota più alta, nei casi di insediamenti in vita senza evidenti strutture legate all'incastellamento e nei casi di insediamenti abbandonati senza l'indicazione di rovine.

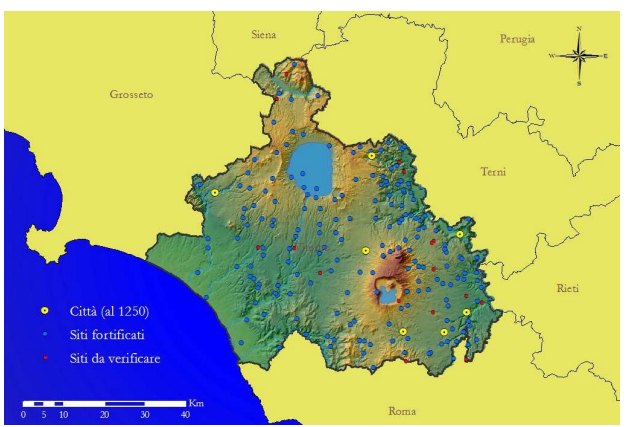

Fig. 2. Totale dei siti georeferenziati attualmente presenti nella banca dati.

Il numero totale dei siti fortificati finora censiti e georeferenziati ammonta a 191, comprendendo quelli che hanno assunto o mantenuto configurazione castrale nel periodo storico in esame, ai quali si aggiungono, per il momento, sedici insediamenti da verificare.

\subsection{Il portale web}

Per la pubblicazione web della banca dati ottenuta si è optato per la creazione di un portale WebGIS. Il formato atlante ci permette di combinare i vantaggi della mappa, in termini di visualizzazione, interrogazione e analisi spaziale della rete, con le prerogative di un catalogo, inteso come insieme ordinato di schede individuali e relazionate, con informazioni di dettaglio sui singoli insediamenti. Il visore offrirà l'accesso a tematismi vettoriali di riferimento, livelli di informazione storica e cartografia di base pertinente, integrata attraverso i servizi WMS pubblicati sul Geoportale Nazionale. 
La rete ricostruita di insediamenti geolocalizzati costituisce, in definitiva, un indice di nomi geografici (gazetteer) e quindi, per favorirne l'accessibilità, si è deciso di utilizzare URIs permanenti per ogni sito, impiegando l'ontologia RDF Linked Places $^{2}$, la quale consente di descrivere e illustrare luoghi del passato, così come di temporalizzare attributi fondamentali, quali: toponimo, geometria, tipologia e relazioni con altri luoghi di rango superiore o inferiore. Questo schema di base, la cui adozione garantisce inoltre la interoperabilità con altri repertori semantici di luoghi geografici, verrà esteso per riflettere le caratteristiche dei dati in esame e i requisiti specifici del progetto. Per la bibliografia, si adotterà invece il formato BIBO (Bibliographic Ontology), standard di riferimento del settore.

\section{Conclusioni}

Il disegno delle caratteristiche e funzionalità del portale web appena descritto costituisce il primo stadio verso la creazione del portale stesso, che occuperà i prossimi mesi di lavoro e per il cui sviluppo restano ancora da definire le soluzioni software da adottare.
La pubblicazione online dei dati, attraverso una piattaforma dinamica, aperta e interoperabile, ci permetterà inoltre di migliorare ed accrescere la banca dati attraverso la collaborazione con la società civile (Toscano 2018), per esempio per includere un apparato fotografico attualmente assente, così come pianificare fasi successive di integrazione e approfondimento attraverso tesi di laurea e di master di studenti dell'Università della Tuscia, ovvero intraprendere iniziative di collaborazione e di studio con altri gruppi di ricerca, per l'ampliamento della rete ai territori limitrofi.

\footnotetext{
Note

${ }^{1}$ La necessità di criteri di posizionamento del punto sulla carta si amplifica con l'avvento dei sistemi GIS, che separano la cartografia dalla sua scala di realizzazione, creando l'illusione della precisione infinita.

${ }^{2}$ Il formato Linked Places, v.1.1, sostituisce il Pelagios Gazetteer Interconnection Format (PGIF) come modello dati RDF sia per i progetti Pelagios (Recogito e Peripleo) che per la World-Historical Gazetteer.
}

\section{Bibliography}

Almagià, R. (1960). Documenti Cartografici dello Stato Pontificio, Città del Vaticano.

Andrews, D. (1978). "Medieval masonry in Northern Lazio: its development and uses for dating", in Blake, H.; Potter, T.W.; Whitehouse, D.B., eds., Papers in Italian Archaeology. The Lancaster Seminar, Oxford, pp. 391-412.

Antonelli, M. (1895). "Una relazione del Vicario del Patrimonio a Giovanni XXII in Avignone". Archivio della R. Società Romana di Storia Patria, XVIII, pp. 447-467.

Battelli, G., ed. (1946). Rationes decimarum Italiae nei secoli XIII e XIV: Latium,Città del Vaticano.

Brunetti Nardi, G., ed. (1972-1981). Repertorio degli scavi e delle scoperte archeologiche nell'Etruria Meridionale, II-III (1966-1970 e 1971-1975), Roma.

Buzzi, C., ed. (1988). Il catasto di S. Stefano di Viterbo, Roma.

Buzzi, C., ed. (1998). Il “Liber quatuor clavium” del Comune di Viterbo, Roma.

Buzzi, C., ed. (2004). Lo Statuto del Comune di Viterbo del 1469, Roma.

Cameron, F.; Clark, G.; Jackson, R.P.J.; Johns, C.M.; Philpot, S.; Potter, T.W.; Shepherd, J.D.; Stone, M.J.; Whitehouse, D.B. (1984). "Il castello di Ponte Nepesino e il confine settentrionale del Ducato di Roma", Archeologia Medievale, XI, pp. 63-147.

Campanari, S. (1856). Tuscania e i suoi monumenti. Documenti istorici, Montefiascone.

Carbonetti Vendittelli, C., ed. (1990). Liber memorie omnium privilegiorum et instrumentorum et actorum communis Viterbii (1283), Roma.

Carocci, S.; Vendittelli, M. (2014). L'origine della Campagna Romana. Casali, castelli e villaggi nel XII e XIII secolo, Roma.

Carusi, E. (1948). Cartulario di S. Maria in Campo Marzio, 986-1199, Roma. 
Chiovelli, R. (2007). Tecniche costruttive medievali. La Tuscia, Roma.

Christie, N., ed. (1991). Three South Etrurian Churches: S. Cornelia, S. Rufina and S. Liberato, London.

Coarelli, F.; Patterson, H., ed. (2004). Mercator placidissimus. The Tiber valley in antiquity. New research in the upper and middle river valley (Rome, 27-28 February 2004), Roma.

Conti, S. (1980). Le sedi umane abbandonate del Patrimonio di S. Pietro in Tuscia, Firenze.

Duncan, G. (1958). "Sutri”, Papers of the British School at Rome, XXVI, pp. 63-134.

Egidi, P. (1901). "Le Cronache di Viterbo scritte da Francesco D’Andrea”, ASRSP, XXIV, pp. 197-252, $299-371$.

Egidi, P. (1906-1907). "L'archivio della Cattedrale di Viterbo"; Bollettino dell'Istituto storico italiano per il Medio Evo e Archivio Muratoriano, 27, pp. 7-382.

Egidi, P., ed. (1910). "Statuti di Castel Fiorentino", in Tomassetti, F.; Federici, V.; Egidi, P. eds., Statuti della Provincia Romana. Vicovaro, Cave, Roccantica, Ripi, Genazzano, Tivoli, Castel Fiorentino, Roma, pp. 303-359.

Egidi, P., ed. (1930). “Gli Statuti Viterbesi del MCCXXXVII-VIII, MCCLI-II e MCCCLVI”, in Federici, V. ed., Statuti della Provincia Romana. S. Andrea in Selci, Subiaco, Viterbo, Roviano, Anagni, Saccomuro, Aspra Sabina, Roma, pp. 27-282.

Fabre, P. (1887). "Un régistre Caméral du Cardinal Albornoz en 1364. Documents pour servir à l'histoire du Patrimonium Beati Petri in Tuscia au quatorzième siècle", Mélanges d'archéologie et d'histoire publiés par l'Ecole française de Rome et d'Athène, VIII, pp. 129-195.

Fabre, P.; Duchesne, L., ed. (1905-1952). Le Liber Censuum de l'Eglise Romaine, Paris.

Fedele, P. (1898-1899). "Carte del monastero dei SS. Cosma e Damiano in Mica Aurea, sec. X e XI", Archivio della R. Società Romana di Storia Patria, XXI, pp. 459-534; XXII, pp. 25-107.

Fedele, P. (1900-1903). “Tabularium Sanctae Mariae Novae ab anno 982 ad anno 1200”, Archivio della R. Società Romana di Storia Patria, XXIII, pp. 171-237; XXIV, pp. 159-196; XXV, pp. 169-209; XXVI, pp. 21-141.

Federici, V. (1899-1900). "Regesto del monastero di San Silvestro in Capite", Archivio della R. Società Romana di Storia Patria, XXII, pp. 213-300; XXXIII, pp. 67-128.

Fronti, D.; Romagnoli, G. (2007). "Indagini archeologiche nel castello di Pian Fasciano (Tuscania). Nota preliminare", in Corneto medievale: territorio, società, economia e istituzioni religiose. Atti del Convegno di studio (Tarquinia, 24-25 novembre 2007), Tarquinia, pp. 421-454.

Frutaz, A.P. (1972). Le carte del Lazio, Città del Vaticano.

Fumi, L. (1884). Codice diplomatico della Città d'Orvieto, Firenze.

Gamurrini, G.F.; Cozza, A.; Pasqui, A.; Mengarelli, R. (1972). Carta archeologica d'Italia (1881-1897). Materiali per l'Etruria e la Sabina, Firenze.

Giorgi, I. (1878). "Il regesto del Monastero di S. Anastasio ad Aquas Salvias", Archivio della R. Società Romana di Storia Patria, I, pp. 49-77.

Güll, P.; Fronti, D.; Romagnoli, G.; Wick, F. (2001). "Viterbo. Indagini archeologiche 1997-1998: nuovi dati per la topografia urbana e la cultura materiale”, Archeologia Medievale, XXVIII, pp. 275-294.

Hartmann, L.M.; Merones, M. ed. (1895-1913). Ecclesiae S. Mariae in Via Lata Tabularium, Vienna.

Istituto Italiano dei Castelli, ed. (1985). Carta dei luoghifortificati del Lazio, Roma.

Jones, G.D.B. (1962-1963). "Capena and the Ager Capenas", Papers of the British School at Rome, XXX, pp. 116207; XXXI, pp. 100-158.

Lanconelli, A. (1992). "Dal «castrum» alla «civitas»: il territorio di Viterbo tra VIII e XI secolo", Società e Storia, 56, pp. 244-266.

Lanconelli, A.; Romagnoli, G. (2018). L'incastellamento nel Lazio, in Augenti, A.; Galetti, P. eds., Incastellamento. Storia e archeologia. A 40 anni da Les structures di Pierre Toubert, Spoleto, pp. 379-389.

Lungo, S. Del. (1998). Il territorio dell'antica diocesi di Orte nella toponomastica archeologica, Orte.

Lungo, S. Del. (1999). La toponomastica archeologica della Provincia di Viterbo, Tarquinia.

Mallet, D.; Whitehouse, D.B. (1967). "Castel Porciano: an abandoned village in the Roman Campagna”, Papers of the British School at Rome, XXXV, pp. 113-146.

Milioni, A. (2002). Viterbo. I (IGM F. 136 I SE Capodimonte, 136 II SE La Rocca, 137 III SO La Commenda, 137 III SO Castel d'Asso, 137 II SE Viterbo, 137 IV SO Montefiascone), Viterbo.

Minicis, E. De., ed. (2003-2011). "Insediamenti rupestri medievali della Tuscia, I-III, RomaMachado, J. (2015), Open data and open science”, in: Albagli, S. ed., Open Science, Open Questions, pp. 189-214. 
Molinari, A. (2010). "Siti rurali e poteri signorili nel Lazio (secc. X-XII)", Archeologia Medievale, 37, pp. 129-142.

Monaci, A. (1904-1905). "Regesto dell'abbazia di Sant'Alessio all'Aventino”, Archivio della R. Società Romana di Storia Patria, XXVII, pp. 351-398; XXVIII, pp. 151-200.

Nardi Combescure, S. (2002). Paesaggi d'Etruria Meridionale. L'entroterra di Civitavecchia dal II al XV secolo d.C., Firenze.

Patterson, H., ed. (2004). Bridging the Tiber. Approaches to regional archaeology in the middle Tiber Valley, London.

Pinzi, C. (1887-1913). Storia della città di Viterbo, Roma.

Potter, T.W. (1979). Storia del paesaggio dell'Etruria Meridionale, Roma 1979.

Potter, T.W.; King, A.C. (1997). Excavations at the Mola di Monte Gelato. A roman and medieval settlement in South Etruria, London.

Romagnoli, G. (2006). Ferento e la Teverina viterbese. Insediamenti e dinamiche del popolamento tra il X e il XIV secolo, Viterbo.

Savignoni, P. (1895). "L'Archivio Storico del Comune di Viterbo", Archivio della R. Società Romana di Storia Patria, XVIII, pp. 5-50.

Scardozzi, G. (2004). Ager Ciminus, Viterbo.

Schiaparelli, A. (1901-1902). "Le carte antiche dell'Archivio Capitolare di San Pietro inVaticano", Archivio della R. Società Romana di Storia Patria, XXIV, pp. 393-496; XXV, pp. 273-354.

Signorelli, G. (1907-1969). Viterbo nella storia della Chiesa, Roma.

Silvestrelli, G. (1914). Città, castelli e terre della regione romana, Città di Castello.

Silvestrelli, G. (1917). "Le chiese e i feudi dell'Ordine dei Templari e dell’Ordine di San Giovanni in Gerusalemme nella Regione Romana", Rendiconti della Reale Accademia dei Lincei. Classe di scienze morali, storiche e filologiche, s. V, XXVI, 5-6, pp. 491-539.

Sommella Mura, A., ed. (1969). Repertorio degli scavi e delle scoperte archeologiche nell'Etruria Meridionale, I (1939-1965), Roma.

Sora, V. (1906). “I Conti d'Anguillara dalla loro origine fino al 1465”, Archivio della R. Società Romana di Storia Patria, XXIX, pp. 397-442.

Supino, P. (1969). La Margherita Cornetana. Regesto dei documenti, Roma.

Theiner, A. (1861-1862). Codex Diplomaticus dominii temporalis S. Sedis, Paris.

Tomassetti, G. (1897). "Del sale e del focatico del Comune di Roma nel Medioevo", Archivio della R. Società Romana di Storia Patria, XX, pp. 313-368.

Tomassetti, G. (1910-1926). La Campagna Romana Antica, Medioevale e Moderna, Roma.

Toubert, P. (1973). Les structures du Latium médiéval. Le Latium méridional et la Sabine du IXe siècle à la fin du XIIe siècle, Roma.

Toscano, M. (2008). "La red de los castillos de la provincia de Viterbo, Italia (siglos X-XV). Primeros resutados de un análisis estadístico-espacial”, in Arqueología y Territorio Medieval, Jáen, pp. 73-89.

Toscano, M. (2018). "Where the researcher cannot get. Open Platforms to collaborate with citizens on cultural heritage research data", in Romero Frías, E.; Bocanegra Barbecho, L. eds., Ciencias Sociales y Humanidades Digitales aplicadas: casos de estudio y perspectivas criticas. Granada, pp. 538-561.

Trifone, B. (1908-1909). "Le carte del Monastero di S. Paolo di Roma dal secolo XI al XV", Archivio della R. Società Romana di Storia Patria, XXXI, pp. 267-313; XXXII, pp. 29-106.

Vallelonga, F. (2012). "Insediamenti fortificati di età medievale in un territorio di confine: l'area dei Monti della Tolfa e la valle del Mignone", in Il ruolo degli oppida e la difesa del territorio in Etruria: casi di studio e prospettive di ricerca, Trento, pp. 173-222.

Vendittelli, M., ed. (2008). Sutri nel medioevo. Storia, insediamento urbano e territorio, Roma.

Wickham, C. (1978-1979). "Historical and topographical notes on early medieval South Etruria", Papers of the British School at Rome, XLVI, pp. 133-179; XLVII, pp. 66-95. 